\title{
Effects of animation retargeting on perceived action outcomes
}

\author{
Sophie Kenny \\ Queen's University \\ Department of Psychology \\ Kingston, Canada K7L 3N6 \\ kenny.s@queensu.ca
}

\author{
Naureen Mahmood \\ Max Planck Institute for Intelligent \\ Systems \\ Tübingen, Germany 72076 \\ nmahmood@tue.mpg.de
}

\author{
Claire Honda \\ Queen's University \\ Department of Psychology \\ Kingston, Canada K7L 3N6 \\ claire.honda@queensu.ca
}

\author{
Michael J. Black \\ Max Planck Institute for Intelligent \\ Systems \\ Tübingen, Germany 72076 \\ black@tuebingen.mpg.de
}

\author{
Nikolaus F. Troje \\ Queen's University \\ Department of Psychology \\ Kingston, Canada K7L 3N6 \\ troje@queensu.ca
}

\begin{abstract}
The individual shape of the human body, including the geometry of its articulated structure and the distribution of weight over that structure, influences the kinematics of a person's movements. How sensitive is the visual system to inconsistencies between shape and motion introduced by retargeting motion from one person onto the shape of another? We used optical motion capture to record five pairs of male performers with large differences in body weight, while they pushed, lifted, and threw objects. Based on a set of 67 markers, we estimated both the kinematics of the actions as well as the performer's individual body shape. To obtain consistent and inconsistent stimuli, we created animated avatars by combining the shape and motion estimates from either a single performer or from different performers. In a virtual reality environment, observers rated the perceived weight or thrown distance of the objects. They were also asked to explicitly discriminate between consistent and hybrid stimuli. Observers were unable to accomplish the latter, but hybridization of shape and motion influenced their judgements of action outcome in systematic ways. Inconsistencies between shape and motion were assimilated into an altered perception of the action outcome.
\end{abstract}

\section{CCS CONCEPTS}

- Computing methodologies $\rightarrow$ Motion capture; • Applied computing $\rightarrow$ Psychology;

\section{KEYWORDS}

retargeting, human animation, animated avatars, shape capture, realism, inconsistency, perception, discrimination, action

\section{ACM Reference format:}

Sophie Kenny, Naureen Mahmood, Claire Honda, Michael J. Black, and Nikolaus F. Troje. 2017. Effects of animation retargeting on perceived action

Permission to make digital or hard copies of part or all of this work for personal or classroom use is granted without fee provided that copies are not made or distributed for profit or commercial advantage and that copies bear this notice and the full citation on the first page. Copyrights for third-party components of this work must be honored.

For all other uses, contact the owner/author(s).

SAP '17, September 16-17, 2017, Cottbus, Germany

(C) 2017 Copyright held by the owner/author(s).

ACM ISBN 978-1-4503-5148-5/17/09.

https://doi.org/10.1145/3119881.3119891 outcomes. In Proceedings of SAP '17, Cottbus, Germany, September 16-17, 2017, 7 pages.

https://doi.org/10.1145/3119881.3119891

\section{INTRODUCTION}

When looking at other people and assessing their actions and behaviour, observers are likely to employ a wealth of prior knowledge about statistical dependencies between body build and kinematic aspects of body movements [Cohen 1981]. Size, weight, relative limb length, and the distribution of mass over the body all influence the way a body moves. Observers have been shown to use shape and motion information in a variety of people perception tasks, including sex discrimination [Mather and Murdoch 1994; Troje 2002, 2008], person identification [Troje et al. 2005; Westhoff and Troje 2007], and action outcome perception [Bingham 1987, 1993; F. de C. Hamilton et al. 2007; Munzert et al. 2010; Runeson and Frykholm 1981, 1983; Shim and Carlton 1997; Shim et al. 2004]. According to the Kinematic Specification of Dynamics principle [Runeson and Frykholm 1981, 1983], observers learn to relate the visually available kinematic patterns of action with the latent forces that would have been exerted to produce the observed motion pattern. Learning occurs through an observer's life-long personal experience with manipulating their natural environment and observing other people in similar situations [Gibson 1979]. Innate predispositions and sensory filter mechanisms may also play a role [Johnson 2006; Troje and Chang 2013].

Given the importance of both body shape and body motion as sources of information for people perception, it is likely that the visual system is sensitive to internal inconsistencies introduced by perturbing the relationship between shape and motion. In computer animation, internal inconsistency can arise during animation retargeting. In this procedure, the recorded motion of a performer is used to animate a humanoid avatar. In the general case, the shapes of the performer and of the avatar might differ considerably [Gleicher 1998]. The aim of animation retargeting procedures is to preserve the motion of the performer such that the high-level features of an action are preserved in the animated avatar [Geng and Yu 2003; Menache 2011]. However, retargeting the motion of a performer onto an avatar with a different body shape is likely to impact perception because the shape of the avatar implies dynamic properties that generally differ from those of the performer [Troje 2013]. 
Research into human action perception has often been conducted using point-light displays [Johansson 1973], which reduce the rendering of a human actor to a relatively small number of dots representing the major joints of the body. The only information about body shape that can be directly obtained from these displays is based on relative limb lengths and the coarse geometry of the articulated array of joints. However, even these reduced displays are sufficient to demonstrate that observers have expectations about the relations between shape and motion when assessing human motion. For example, observers seem to be able to use knowledge of the physically determined relationship between walking stride length and stride frequency to appropriately scale the size of an animal [Jokisch and Troje 2003]. Naturalness ratings of point-light walkers presented at a veridical, sped-up, or slowed-down playback speed, betray the visual system's sensitivity to inconsistency: observers rate the veridical playback speeds of point-light walkers as most natural [Troje and Lau 2012]. In another experiment, Klüver, Hecht, \& Troje [Klüver et al. 2016] demonstrated that exchanging the shape and motion information between pairs of point-light walkers directly resulted in lower attractiveness for hybrid walkers, compared to consistent walkers.

Information from movement kinematics is often sufficient to support the perception of action outcomes. For example, throwing distance of invisible objects can be inferred from the arm kinematics of point-light displays [Munzert et al. 2010; Runeson and Frykholm 1983; Zhu and Bingham 2014]. For other actions, body shape is more likely to influence how kinematic information is interpreted by the visual system. For instance, although kinematics support the perception of a lifted weight [Bingham 1987; F. de C. Hamilton et al. 2007; Shim and Carlton 1997], the weight of the performer may modulate how kinematics are used. According to Runeson \& Frykholm [Runeson and Frykholm 1981], light performers who lift increasingly heavy boxes adjust their posture to stabilize themselves as a counterweight to the box, while heavier performers do not require postural adjustments as they have a more stable base of support. This evidence highlights the action-specificity in the way shape and motion are integrated in the perception of action outcomes.

The above mentioned studies all employed point-light displays. Similar work with more realistic animations that provide a richer visualization of individual body shape is rare. The reason is that such work requires accurate measurements of body kinematics and full 3D body shape from individual actors. Traditionally, body kinematics are sampled with optical motion capture technology. Body shape can be assessed with 3D whole body scanners. More recently, however, Loper and colleagues [Loper et al. 2014] have devised MoSh, a technique that fits a parametric model of body shape and pose directly to 3D motion capture markers. The body shape is constrained to lie in the space of human body shapes which is represented by a SCAPE body model [Anguelov et al. 2005] learned from approximately 4000 3D body scans [Hirshberg et al. 2012]. MoSh achieves reconstruction errors that are smaller than $1 \mathrm{~cm}$ (mean distance between true and estimated body shape). While this is not sufficient to reconstruct idiosyncratic details of hands and face, the system recovers individual 3D body shape and
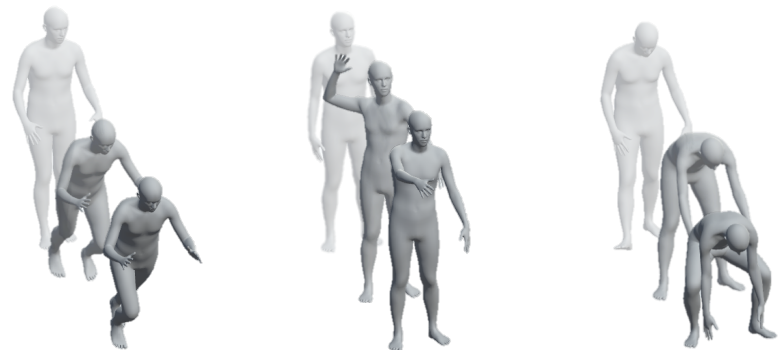

Figure 1: Realistic human animations of performers recorded while pushing, throwing, and lifting objects.

its dependencies on pose with a high degree of accuracy [Loper et al. 2014].

Does a disruption of the natural relationship between body shape and body motion impact perception of action outcomes? In the current study, we analyze the effects of stimulus inconsistency introduced by retargeting motion between people with different body masses. We test whether observers are able to explicitly discriminate consistent renderings from hybrids, and we measure the effects of retargeting on perception of action outcomes. We study the effect of inconsistency for actions in which shape and motion information contribute differently to the action outcome. We studied the perception of three action outcomes that rely on different relative contributions of shape and motion characteristics of the animated avatars: pushing training sleds (shape and motion), throwing a light object (mostly motion), and lifting a box. These actions occur commonly in video games and are representative for transitive actions that involve object manipulations.

\section{STIMULI}

We used optical motion capture to record action performance of 10 male volunteers from Queen's University, Canada, who we classified in two body weight categories. Five light performers weighed on average $69.9 \mathrm{~kg}(S D=6.55 \mathrm{~kg})$, and five heavy performers weighed on average $108 \mathrm{~kg}(S D=12.8 \mathrm{~kg})$. We created five heightmatched pairs by maximizing body weight differences between light and heavy performers. We recruited only male performers to focus the variance among our stimuli on weight differences and avoid confounding them with sex-related interactions.

Performers were recorded performing three actions: pushing a training sled weighing between $23 \mathrm{~kg}$ to $123 \mathrm{~kg}$, in $20 \mathrm{~kg}$ increments; throwing beanbags weighing $200 \mathrm{~g}$ towards targets located $2 \mathrm{~m}$ to $6 \mathrm{~m}$ away, in $1 \mathrm{~m}$ increments; and lifting boxes weighing from $1 \mathrm{~kg}$ to $11 \mathrm{~kg}$, in $2 \mathrm{~kg}$ increments (Figure 1).

Next, we used the MoSh algorithm [Loper et al. 2014] to obtain correlated estimates for body shape and body motion for each performer, each action, and each object weight and throwing distance. Body shape and body motion were used to create consistent and inconsistent animated avatars. Consistent animated avatars were created by combining shape and motion from a single performer, while inconsistent animated avatars were created by hybridizing shape and motion from a pair of height-matched performers, one from each body weight category (Figure 2). 


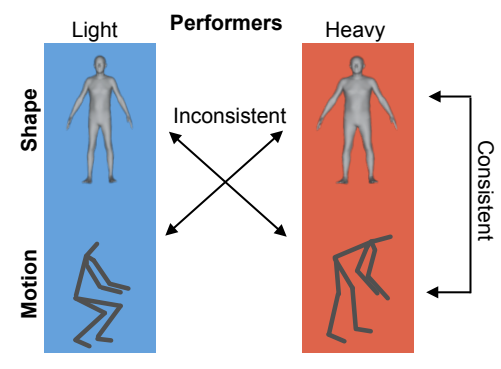

Figure 2: Consistency of shape and motion information was manipulated through selection of the source of the shape and motion estimates. Consistent stimuli were animated avatars whose shape and motion were taken from the same performer, and inconsistent stimuli were animated avatars whose shape and motion were taken from were taken from height-matched performers with highly dissimilar body weights.

The combination of 10 actors, 6 different weights, and both consistent and hybrid versions resulted in a stimulus set that consisted of 120 stimuli for the box lifting task and 120 stimuli for the sled pushing task. Since there were only 5 different distances in the throwing task, this resulted in a stimulus set of 100 different throwing stimuli. See the supplementary video for examples of the stimuli.

\section{EXPERIMENT 1 : ACTION OUTCOMES}

\subsection{Participants}

Sixty-three volunteers from Queen's University, 42 women and 21 men, were randomly assigned to observe either throwing, pushing, or lifting such that there were 21 participants in each group. Participants were between 17 and 34 years of age $(M=21.4$ years old, $S D=3.97)$. They were tested individually and reported normal- or corrected-to-normal vision.

\subsection{Procedure}

In order to familiarize participants with the scenarios used in the experiment, they were shown typical examples of the items that were used during the motion capture session: the box that was lifted, the sled that was pushed, and the beanbag that was thrown, as well as the demarcated distances of the throwing task. However, they were not informed about the numerical values of the real weights and distances that would be presented. Participants were then seated and outfitted with a head-mounted display. The headset simulated a three-dimensional stereoscopic virtual reality testing room. Real-time head tracking was used to update rendering such that it realistically reflected observer movements and allowed the participant to actively explore the scene by moving and turning their head. For the head-mounted display we used Oculus Rift DK2, runtime version 0.8.0.0. The experimental program was written using Unity3D, version 5.1.4, and the Unity Oculus Plugin.

Participants viewed either the pushing, throwing, or lifting stimulus sets. Stimuli were presented one at a time in a randomized order. All stimuli were rotated 20 degrees counterclockwise from facing the participant. The pushing and lifting actions first appeared

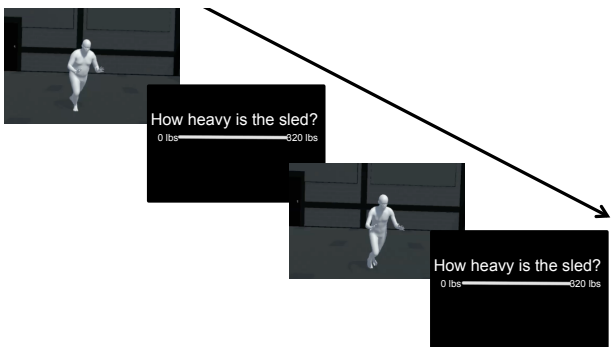

Figure 3: Following the presentation of each stimulus, participants indicated the perceived action outcomes using a continuous response scale. Consistent and inconsistent stimuli were randomly presented. Actions were varied as betweensubjects factors.
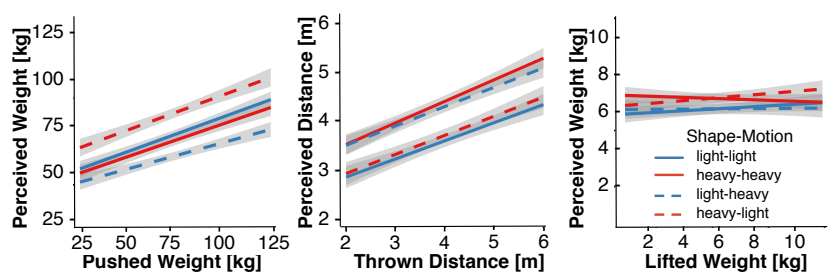

Figure 4: Regression lines representing the relationship between perceived and real weights and distances. The shaded areas represent \pm 1 SEM

at a distance of $6 \mathrm{~m}$ ahead of the observers, and the throwing action at $4 \mathrm{~m}$, such that all three actions concluded at a distance of $4 \mathrm{~m}$ ahead of the observer. Participants could move their head, but this was not required to maintain the stimuli within view. After presentation of each stimulus, participants were prompted to input the perceived action outcomes on a continuous rating scale (Figure 3). The scales ranged from $0 \mathrm{lbs}$ to $24 \mathrm{lbs}$ for the lifted weight, from $0 \mathrm{lbs}$ to $320 \mathrm{lbs}$ for the weight of the pushed sled, and from $1 \mathrm{~m}$ to $7 \mathrm{~m}$ for the distance of the thrown beanbag. Note that perceived weights were reported in pounds $(1 \mathrm{lbs}=0.454 \mathrm{~g})$ because participants in pilot studies indicated a greater familiarity with imperial units of weight. No performance feedback was provided. The procedure was repeated twice with a new random presentation order for the second testing block. There was a short break introduced between blocks. The whole testing session lasted about $80 \mathrm{~min}$, or about $60 \mathrm{~min}$ for the participants in the throwing group.

\subsection{Results and discussion}

The contributions of shape, motion, and their interaction varied between the three tested actions (Figure 4). We performed independent 2 (shape) $\times 2$ (motion) repeated-measures ANOVAs on perceived object weights for each of the different actions. Levels of both factors indicated whether the factor was based on data from a heavy or from a light person.

3.3.1 Pushing. There was a significant effect of shape, $F(1,20)=$ $43.4, p<.001, \eta_{p}^{2}=.684$. Avatars with the shape of a heavy performer were perceived as pushing heavier sleds $(M=76.2 \mathrm{~kg}$, 
$S E M=2.83 \mathrm{~kg})$ than avatars with light performer shapes $(M=$ $66.2 \mathrm{~kg}, S E M=2.40 \mathrm{~kg})$. There was also a significant effect of motion, $F(1,20)=103, p<.001, \eta_{p}^{2}=.838$. Avatars that were animated with the motion of a heavy performer were perceived as pushing lighter sleds $(M=64.4 \mathrm{~kg}, S E M=2.52 \mathrm{~kg})$ than avatars with light performer motions $(M=78.0 \mathrm{~kg}, S E M=2.68 \mathrm{~kg})$. Finally, there was also a significant interaction between shape and motion, $F(1,20)=18.0, p<.001, \eta_{p}^{2}=.486$. Observers perceived similar sled weights for the two conditions in which avatar shape and motion were taken from consistent sources of information (i.e. light performer shape matched with light performer motion and heavy performer shape matched with heavy performer motion), paired $t$-test, $t(20)=-1.99, p=.061$. However, hybrid avatars resulted in lighter perceived sled weights if stimuli were created from a combination of light performer shapes and heavy performer motions $(M=60.3 \mathrm{~kg}, S E M=2.46 \mathrm{~kg})$, and resulted in heavier perceived weights if stimuli were created from heavy performer shapes and light performer motions $(M=83.9 \mathrm{~kg}, S E M=3.10 \mathrm{~kg})$. Note that ratings for the consistent performers were very similar, no matter whether they were light or heavy. Observers apparently take body shape of performers into account when interpreting their poses and kinematics. The observed interaction effect demonstrates that the inconsistent combinations modify perception of sled weight beyond the independent, additive contributions of shape and motion.

The slopes of the regression lines (Figure 4) were influenced by both shape and motion. A 2 (shape) x 2 (motion) repeatedmeasures ANOVA on the slope values a significant effect of shape, $F(1,20)=10.1, p=.005, \eta_{p}^{2}=.335$, with heavy body shapes resulting in steeper perceived slopes $(M=0.374, S E M=0.030)$ to those of light body shapes $(M=0.324, S E M=0.324)$. There was also a significant effect of motion, $F(1,20)=10.2, p=.005, \eta_{p}^{2}=.338$, with the light body motion resulting in steeper perceived slopes $(M=0.384, S E M=0.035)$ to those of heavy body motion $(M=$ $0.314, S E M=0.029)$. The interaction between shape and motion was not significant, $F(1,20)=0.335, p=.569, \eta_{p}^{2}=.016$. The effects of shape and motion on slope are consistent with studies of the kinematics of sled pushing, that suggest that the body weight of an individual will strongly modulate the kinematics of the pushing motion [Hoffman 2014], as well as with the KSD principle that suggests that the motion of light individuals is more strongly impacted by changes in object weight than the motion of heavy individuals [Runeson and Frykholm 1981, 1983].

3.3.2 Throwing. There was a strong effect of motion, $F(1,20)=$ $2.20, p<.001, \eta_{p}^{2}=.811$. Avatars that were animated with the motion of a heavy performer were perceived as throwing farther $(M=$ $4.35 \mathrm{~m}, S E M=0.115 \mathrm{~m}$ ) than avatars animated with motion from light performers $(M=3.59 \mathrm{~m}, S E M=0.140 \mathrm{~m})$. There was also a small, yet significant effect of shape, $F(1,20)=9.97, p=.005, \eta_{p}^{2}=$ .333. Avatars with the shape of a heavy performer were perceived as throwing slightly farther $(M=4.03 \mathrm{~m}, S E M=0.127 \mathrm{~m})$ than avatars based on light performer shapes $(M=3.91 \mathrm{~m}, S E M=$ $0.112 \mathrm{~m})$. There was no interaction between shape and motion, $F(1,20)=0.847, p=.368, \eta_{p}^{2}=.041$. This is consistent with previous literature on point-light displays. Distance estimation from arm kinematics can be achieved in the absence of an explicit representation of the rest of the body [Munzert et al. 2010].
The slopes of the regression lines (Figure 4) were not influenced by shape or motion. A 2 (shape) x 2 (motion) repeated-measures ANOVA on the slope values revealed no significant effect of shape, $F(1,20)=2.59, p=.123, \eta_{p}^{2}=.115$, with heavy body shapes resulting in similar perceived slopes $(M=0.437, S E M=0.029)$ to those of light body shapes $(M=0.407, S E M=0.030)$. There was also no significant effect of motion, $F(1,20)=0.116, p=.775, \eta_{p}^{2}=.006$, with the heavy body motion resulting in similar perceived slopes $(M=0.426, S E M=0.031)$ to those of light body motion $(M=0.418$, $S E M=0.030)$. The interaction between shape and motion was not significant, $F(1,20)=3.40, p=.080, \eta_{p}^{2}=.145$. These results also agree with the suggestion that perception of object throwing distances is not modulated by performer body shape [Munzert et al. 2010].

3.3.3 Lifting. There was a significant effect of shape, $F(1,20)=$ $27.9, p<.001, \eta_{p}^{2}=.582$. Avatars with heavy performer shapes were perceived to lift heavier boxes $(M=5.90 \mathrm{~kg}, S E M=0.780 \mathrm{~kg})$ than avatars with light performer shapes $(M=5.44 \mathrm{~kg}, S E M=$ $0.168 \mathrm{~kg})$. There was no significant effect of motion, $F(1,20)=$ $0.290, p=.596, \eta_{p}^{2}=.014$. There was also no interaction between shape and motion, $F(1,20)=0.570, p=.459, \eta_{p}^{2}=.028$. All effects on perceived box weight seemed to be carried by the shape of the performer.

However, further examination of the results provided evidence that observers recovered box weights only very poorly, as evidenced by the shallow slopes of the regression lines (Figure 4). There is weak evidence that motion from light performers helps observers perform the task. A 2 (shape) x 2 (motion) repeatedmeasures ANOVA on the slope values revealed no significant effect of shape, $F(1,20)=0.181, p=.675$. However, there was a significant effect of motion, $F(1,20)=8.46, p=.009, \eta_{p}^{2}=.297$. Stimuli created with light performer motions resulted in slightly steeper slopes $(M=0.073, S E M=0.018)$ to those of stimuli created with heavy performer motions $(M=-0.006$, $S E M=0.015)$. The interaction between shape and motion was not significant, $F(1,20)=3.31, p=.084$. The slight effect of motion on slope is consistent with the idea that it is easier to perceive differences in box weights with light body motion than with heavy body motion. This would be consistent with Runeson's [Runeson and Frykholm 1981] hypothesis that heavy individuals will not need to significantly adjust their posture when lifting weights, because of the high stability of their center of moment during the box lift. In contrast, light performers must change their posture more for higher weights to account for the destabilization incurred by the box lift, which might be a helpful cue in recovering box weights. However, the slope values were close to zero, and the above interpretation would require validation in further experiments that use heavier box weights that contribute more strongly to postural readjustments.

Note that the action outcome perception task was relatively difficult, as evidenced by the relatively shallow slopes illustrated in Figure 4 . The virtual reality task, although ecologically valid, may be more difficult than other methods used to study similar topics. For instance, past research with point-light displays has shown that fixation patterns are oriented towards task-relevant, motion rich areas of visual displays [Saunders et al. 2010]. However, even 
in simple tasks such as gender identification from walking stimuli, eye gaze fixation patterns can differ significantly depending on the rendering of the stimulus: fixations on point-light displays are mostly towards the hips and feet [Saunders et al. 2010], while fixations on animated avatars are mostly on the upper body and faces [Ennis et al. 2015]. The explicit surface of the avatars might influence which regions of the body the observer attends to, incidentally reducing the ability to extract the relevant information. For example, in studies of detection of cloned motion, high variability in the surface appearance of avatars appears to distract from the informative walking motion, in turn increasing the difficulty of a matrix search task [McDonnell et al. 2008]. Technological limitations of head-mounted displays may also alter low-level perceptual information [Renner et al. 2013].

In summary, we found that the visual system uses knowledge about the relations between body shape and motion information to infer action outcomes. However, the effects of shape and motion as well as their interaction differed across the three actions that we evaluated. While perception of throwing distances most strongly depended on the source of the motion estimate, perception of sled weight depended on both components and was also strongly influenced by their interaction.

The results from Experiment 1 suggest that the visual system uses action-specific knowledge of shape and motion information to perceive action outcomes and that it responds to recombining shape and motion in the process of retargeting. In Experiment 2, we asked if the hybrid stimuli are perceived as inconsistent or otherwise flawed, or if attributing detected changes to apparent changes in the nature of the manipulated objects and the action outcome assimilates them into a new, consistent percept that looks as natural as the original ones that contributed to the hybrid.

\section{EXPERIMENT 2: DISCRIMINATION}

\subsection{Participants}

Twenty-four new volunteers from Queen's University were recruited. Participants were 15 females and 9 males, and were between 18 and 24 years of age $(M=19.4$ years old, $S D=1.58$ years old). Participants were tested individually and reported normal or corrected-to-normal vision. None of them had participated in Experiment 1.

\subsection{Procedure}

The stimuli were the same five height-matched pairs of performers described in Section 2, but we used only one recording for each of the three actions, the $11 \mathrm{~kg}$ box lifts, $123 \mathrm{~kg}$ sled pushes, and $6 \mathrm{~m}$ throws. With 10 performers and only three action clips for each, we used a total of 30 veridical, consistent stimuli, and their respective hybridized versions.

We informed participants that they would be seeing performers lifting invisible boxes, pushing invisible sleds, and throwing invisible beanbags. We also told them that ten different male actors had been recorded for the purpose of this study. Participants were informed that they would see pairs of stimuli, always consisting of both a consistent animated avatar and of a hybrid avatar made with the body of one performer and the motion of another. They were instructed to identify which of the two stimuli was the hybrid.

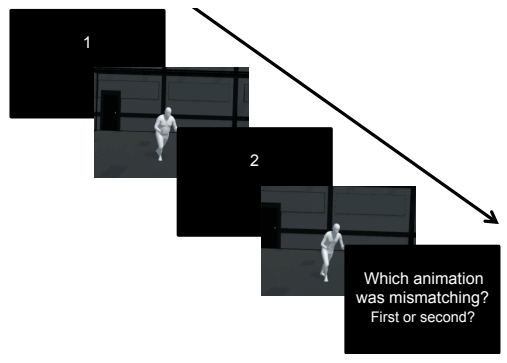

Figure 5: Following the presentation of each consistentinconsistent pair, participants indicated which of the two stimuli they believed was the retargeted hybrid. Participants indicated their response by key press. Actions were randomly presented within a block. In one block, the shape component remained the same between the two stimuli, and in the other block, the motion component remained the same. Block order was counterbalanced.

The two stimuli were presented sequentially, separated by a $500 \mathrm{~ms}$ interstimulus interval. After presentation of each pair, participants pressed a key to identify whether the first or the second stimulus was the hybrid (Figure 5).

Each stimulus pair was presented twice for a total of 60 trials per block. Two blocks were run, for a total of 120 trials. In one block, the same shape was used for both stimuli of a pair, and the motion differed. In the other block, the same motion was used for both stimuli of a pair, and the shape differed. Participants were aware of this manipulation. The order of the two blocks was counterbalanced across participants.

\subsection{Results and discussion}

The results of the inconsistency discrimination task are presented in Figure 6. When asked to discriminate between inconsistent and consistent stimuli, participants' performance remained at chance level. One-sample, two-tailed $t$-tests comparing the correct response rates were not statistically significant for box lifts, $t(23)=1.69, p=$ .105 , sled pushes, $t(23)=1.74, p=.095$, and object throws, $t(23)=$ $0.100, p=.834$.

A two-way repeated-measures ANOVA with actions and blocks as factors was conducted on the correct response rates. There was no main effect of action: participants discriminated between inconsistent and consistent stimuli at an equally low rate across all actions, $F(2,46)=1.76, p=.184, \eta_{p}^{2}=.071$. There was also no main effect of blocks: participant performance was the same when either the shape or the motion information had been manipulated within a pair of stimuli, $F(1,23)=0.141, p=.711, \eta_{p}^{2}=.006$. There was also no interaction between the two factors, $F(2,46)=0.892, p=$ $.417, \eta_{p}^{2}=.037$.

The results of the discrimination experiment support the interpretation that, while the visual system is sensitive to the manipulations involved with retargeting an animation onto another body, the manipulation is not interpreted as inconsistency of animation. Rather, the effect of hybridized animations is reinterpreted as a change in dynamics and attributed to the manipulated objects and the action outcome. 


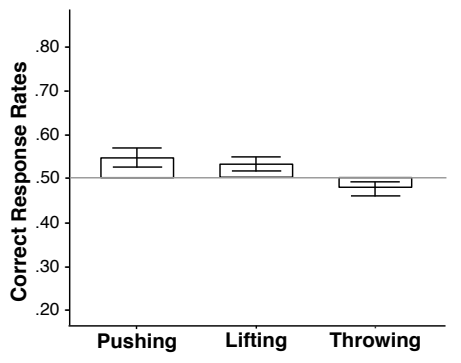

Figure 6: Correct response rates across 24 participants for the inconsistent stimulus discrimination task. Pairs of corresponding inconsistent and consistent stimuli were presented sequentially, and participants pressed a key to indicate whether the first or second stimulus was mismatched. Error bars represent \pm 1 SEM.

\section{DISCUSSION AND CONCLUSIONS}

The visual system has undeniably access to vast knowledge regarding the role of shape and motion information in action production. Past research has shown that knowledge about relationships between shape and motion are used for a number of different person perception tasks [Bingham 1987, 1993; F. de C. Hamilton et al. 2007; Mather and Murdoch 1994; Munzert et al. 2010; Runeson and Frykholm 1981, 1983; Shim and Carlton 1997; Shim et al. 2004; Troje 2002, 2008; Troje et al. 2005]. Most of this previous work, however, was conducted using point-light displays. Here, we are using realistic avatar renderings, which combine a person's body kinematics with detailed individual body shape and therefore provide the observer with much more information and realism about a person's individual appearance. Our experimental results suggest that when the performers that contribute to a hybrid are matched for height, but have very different body mass and body mass distributions, hybridization results in changes in the perceived dynamics of the actions, but are not identified as inconsistent animations.

Our results seem to contrast previous work that used point-light displays of walking people and showed that inconsistency between the geometry of the body and its kinematics reduced attractiveness of walkers [Klüver et al. 2016; Troje and Lau 2012]. Unlike the movements that we used in the current study, walking is not a transitive action and does not involve an object. In our study, the objects and their perceived properties provide additional degrees of freedom that allow the observer to come up with a new valid and consistent re-interpretation of the observed event. It is the objects and their properties that absorb the changes introduced to the stimulus by combining shape and motion from different people. The fact, that the objects themselves were invisible in our study might have given the observer even more freedom to reinterpret the observed events.

If that interpretation is valid, then our results would be specific to transitive actions. Locomotion actions as walking, running, jumping, and many other activities that do not involve the direct manipulation of objects may be more likely to reveal to the observer inconsistencies that result from retargeting. Similar reasoning has been invoked by researchers who manipulated motion patterns during throwing actions [Zhu and Bingham 2014]. The authors demonstrated that these movements can be deformed to a very large extent before the visual system starts to interpret them as unnatural. The visual system may seek valid interpretations of the world whenever possible. That may be easier for transitive actions, which offer more opportunities to discount for inconsistencies introduced in the retargeting process.

In conclusion, the results have important implications for retargeting in computer animation. It seems that the human visual system, while knowledgeable about the natural relations between kinematics and body shape in human motion, is willing to accept reinterpretations of retargeted animations, if possible at all. However, the animator has to be aware of potential changes in the perception of manipulated objects, which may have unintended effects on the narrative of an event. In our experiments, the manipulated objects were invisible, which provided additional freedom for the imagination of the observer to adjust their properties to fit the scene. Even if they were visible, some of their properties (such as the weight) may not be obvious. However, in other situations, even renderings of transitive actions may constrain object properties to a point at which they do not provide enough freedom for a consistent re-interpretation that avoids a sense of inconsistency.

\section{ACKNOWLEDGMENTS}

The authors would also like to thank the anonymous referees for their valuable comments and helpful suggestions. This work was supported by a NSERC Discovery grant and the Humboldt Research Award to NFT, as well as a NSERC Alexander Graham-Bell Graduate Scholarship to SK. Additional travel funds were provided to SK by the International Research and Training Grant "The Brain in Action" (CREATE/DFG).

\section{REFERENCES}

Dragomir Anguelov, Praveen Srinivasan, Daphne Koller, Sebastian Thrun, Jim Rodgers, and James Davis. 2005. SCAPE: Shape completion and animation of people. In ACM Transactions on Graphics (TOG), Vol. 24. 408-416.

Geoffrey P. Bingham. 1987. Kinematic form and scaling: Further investigations and the visual perception of lifted weight. Fournal of Experimental Psychology: Human Perception and Performance 13, 2 (May 1987), 155-177.

Geoffrey P. Bingham. 1993. Scaling judgments of lifted weight: Lifter size and the role of the standard. Ecological Psychology 5, 1 (January 1993), 31-64.

Claudia E. Cohen. 1981. Person categories and social perception: Testing some boundaries of the processing effect of prior knowledge. Fournal of Personality and Social Psychology 40, 3 (January 1981), 441.

Cathy Ennis, Ludovic Hoyet, and Carol O'Sullivan. 2015. Eye-tracktive: Measuring attention to body parts when judging human motions. In Eurographics. 47-40.

Antonia F. de C. Hamilton, Daniel W. Joyce, J. Randall Flanagan, Chris D. Frith, and Daniel M. Wolpert. 2007. Kinematic cues in perceptual weight judgement and their origins in box lifting. Psychological Research 71, 1 (January 2007), 13-21.

Weidong Geng and Gino Yu. 2003. Reuse of motion capture data in animation: A review. Computational Science and Its Application. ICCSA 2003. Lecture Notes in Computer Science 2669, 620-629.

James J. Gibson. 1979. The Ecological Approach to Visual Perception. Boston: Houghton Mifflin.

Michael Gleicher. 1998. Retargetting motion to new characters. Proceedings of the 25th Annual Conference on Computer Graphics and Interactive techniques, 33-42.

David A. Hirshberg, Matthew Loper, Eric Rachlin, and Michael J. Black. 2012. Coregistration: Simultaneous alignment and modeling of articulated 3D shape, A. Fitzgibbon, S. Lazebnik, P. Perona, Y. Sato, and C. Schmid (Eds.). European Conference on Computer Vision (2012), 242-255.

James Jr. Hoffman. 2014. An investigation of the sled push exercise: Quantification of work, kinematics, and related physical characteristics. Ph.D. Dissertation. Sport Physiology and Performance, East Tennessee State University.

Gunnar Johansson. 1973. Visual perception of biological motion and a model for its analysis. Perception \& Psychophysics 14, 2 (June 1973), 201-211. 
Mark H. Johnson. 2006. Biological motion: A perceptual life detector? Current Biology 16, 10 (May 2006), R376-R377.

Daniel Jokisch and Nikolaus F. Troje. 2003. Biological motion as a cue for the perception of size. Journal of Vision 3, 4 (May 2003), 252-264.

Malte Klüver, Heiko Hecht, and Nikolaus F. Troje. 2016. Internal consistency predicts attractiveness in biological motion walkers. Evolution and Human Behavior 37, 1 (January 2016), 40-46.

Matthew Loper, Naureen Mahmood, and Michael J. Black. 2014. MoSh: Motion and shape capture from sparse markers. ACM Transactions on Graphics (TOG) 33, 6, $1-10$.

George Mather and Linda Murdoch. 1994. Gender discrimination in biological motion displays based on dynamic cues. Proceedings of the Royal Society of London B: Biological Sciences 258, 1353 (December 1994), 273-279.

Rachel McDonnell, Michaél Larkin, Simon Dobbyn, Steven Collins, and Carol O'Sullivan. 2008. Clone attack! Perception of crowd variety. In ACM Transactions on Graphics, Vol. 27. 1-8.

Alberto Menache. 2011. Understanding Motion Capture for Computer Animation and Video Games. (2nd ed.). Morgan Kaufmann Publishers.

Jörn Munzert, Tanja Hohmann, and Ernst-Joachim Hossner. 2010. Discriminating throwing distances from point-light displays with masked ball flight. European Journal of Cognitive Psychology 22, 2 (January 2010), 247-264.

Rebekka S. Renner, Boris M. Welichkovsky, and Jens R. Helmert. 2013. The perception of egocentric distances in virtual environments - A review. ACM Computing Surveys (CSUR) 46, 2 (November 2013), 1-40.

Sverker Runeson and Gunilla Frykholm. 1981. Visual perception of lifted weights. Journal of Experimental Psychology: Human Perception and Performance 7, 4 (August 1981), 733-740.

Sverker Runeson and Gunilla Frykholm. 1983. Kinematic specification of dynamics as an informational basis for person-and-action perception: Expectation, gender recognition, and deceptive intention. fournal of Experimental Psychology: General 112, 4 (December 1983), 585-615.

Daniel R. Saunders, David K. Williamson, and Nikolaus F. Troje. 2010. Gaze patterns during perception of direction and gender from biological motion. fournal of Vision 10, 11 (September 2010), 1-10.

Jaeho Shim and Les G. Carlton. 1997. Perception of kinematic characteristics in the motion of lifted weight. fournal of Motor Behavior 29, 2 (March 1997), 131-146.

Jaeho Shim, Les G. Carlton, and Jitae Kim. 2004. Estimation of lifted weight and produced effort through perception of point-light display. Perception 33, 3 (March 2004), 277-291.

Nikolaus F. Troje. 2002. The little difference: Fourier based gender classification from biological motion. In Dynamic Perception, R. P. Würtz and M. Lappe (Eds.). AKA Press, Berlin, 115-120.

Nikolaus F. Troje. 2008. Retrieving information from human movement patterns. In Understanding Events: How Humans See, Represent, and Act on Events, T. F. Shipley and J. M. Zacks (Eds.). Oxford University Press, 308-334.

Nikolaus F. Troje. 2013. What is biological motion? Definition, stimuli and paradigms. In Social Perception: Detection and Interpretation of Animacy, Agency and Intention, M. D. Rutherford and V. A. Kuhlmeier (Eds.). MIT Press, 13-36.

Nikolaus F. Troje and Dorita H. F. Chang. 2013. Shape-independent processes in biological motion perception. In People Watching: Social, Perceptual, and Neurophysiological Studies of Body Perception, K. L. Johnson and M. Shiffrar (Eds.). Oxford University Press, 82-100.

Nikolaus F. Troje and Stephanie Lau. 2012. Perceived naturalness of human motion depends on internal consistency. Journal of Vision 12, 9 (2012), 466.

Nikolaus F. Troje, Cord Westhoff, and Mikhail Lavrov. 2005. Person identification from biological motion: Effects of structural and kinematic cues. Perception \& Psychophysics 67, 4 (February 2005), 667-675.

Cord Westhoff and Nikolaus F. Troje. 2007. Kinematic cues for person identification from biological motion. Perception \& Psychophysics 69, 2 (February 2007), 241-253.

Qin Zhu and Geoffrey P. Bingham. 2014. Seeing where the stone is thrown by observing a point-light thrower: Perceiving the effect of action is enabled by information, not motor experience. Ecological Psychology 26 (October 2014), 229-261. 